\title{
Estudio comparativo del impacto de la media y varianza del tiempo de entrega y de la demanda en el costo del inventario
}

\section{A Comparative Study About the Impact of the Mean and Variance of Lead Time and Demand on the Inventory Cost}

\author{
Izar-Landeta Juan Manuel \\ Universidad del Centro de México, San Luis Potosí \\ Departamento de Investigación \\ Correo:jmizar@hotmail.com \\ Ynzunza-Cortés Carmen Berenice \\ Universidad Tecnológica de Querétaro \\ Mantenimiento Industrial \\ Correo:ynzunzaber@hotmail.com
}

\author{
Castillo-Ramírez Arturo \\ Universidad Autónoma de San Luis Potosí \\ Facultad de Ingeniería \\ Correo:arturo.castillo@uaslp.mx \\ Hernández-Molinar Raúl \\ Universidad Autónoma de San Luis Potosí \\ Facultad de Ingeniería \\ Correo: raul.hernandez@uaslp.mx
}

Información del artículo: recibido: noviembre de 2015, reevaluado: enero de 2016, aceptado: mayo de 2016

\section{Resumen}

Existen muchas opiniones del impacto de la media y desviación estándar del tiempo de entrega, al igual que de la demanda, en el costo del inventario. Este estudio presenta un análisis de dos artículos, uno con distribución de probabilidad normal y el otro con distribución uniforme, para diferentes valores del nivel de servicio, identificando cuáles variables tienen mayor influencia en dicho costo. Para el producto con distribución normal, las dos variables significativas por su impacto son, en primer lugar, la desviación estándar del tiempo de entrega y, en segundo lugar, la media de la demanda. Para el artículo con distribución uniforme, resultaron significativas las cuatro variables, la media y desviación estándar tanto de la demanda como del tiempo de entrega, en donde las de mayor impacto son la media de la demanda y la desviación estándar del tiempo de entrega, lo que varió con el nivel de servicio que se manejó. En todos los casos, el costo del inventario varió linealmente con la desviación estándar del tiempo de entrega. Los resultados obtenidos no cambian con la estructura de costos del inventario, en particular el de faltantes y de mantenimiento del inventario, que son los que definen el nivel de servicio. Para el manejo del inventario se recomienda seleccionar un proveedor que ofrezca un tiempo de entrega razonable con mínima variabilidad.

\section{Descriptores:}

- inventario de seguridad

- tiempo de adelanto

- costo del inventario

- punto de reorden

- demanda del tiempo de entrega 


\begin{abstract}
There are many perspectives about the impacts of the mean and standard deviation of both the demand and the lead-time over the inventory cost. Based upon statistical information, this study presents the results of two analyzed items: one with normal probability distribution and the other with uniform distribution for different values of the level of service, identifying which of the afore mentioned variables have greater influence on the cost. For the item with normal distribution, the variables with the greater impact were the standard deviation of the lead-time and, to a lesser extent, the average demand. For the article with uniform distribution, the four variables, i.e. the mean and standard deviation of both the demand and the lead-time were found to have an impact, with the average demand and the standard deviation of the lead time accounting for the greatest impacts, which also varied according to the level of service used. In all cases analyzed, the cost of inventory varied linearly with the standard deviation of lead time. The results are not affected by the structure of the inventory costs, including stock out and inventory maintenance, which are the two variables defining the level of service. In order to have an optimum inventory management it is recommended to select a provider offering reasonable lead-time with minimum variability.
\end{abstract}

\section{Introducción}

Actualmente, en la mayoría de las naciones, las Pymes producen gran parte del producto interno bruto, por ello es necesario manejar sus recursos de manera eficiente. Este estudio aborda el tema del manejo de los inventarios, que muchas veces representan un porcentaje importante del activo de las organizaciones, por lo que deben administrarse correctamente.

El tema de los inventarios ha dado lugar a muchos trabajos de investigación por parte de académicos y empresarios, buscando que cumplan funciones a un costo mínimo.

La función primordial del inventario es la de proveer artículos de manera suficiente, de modo que si la demanda aumenta no haya faltantes, ya que representarían pérdidas en venta y muy posiblemente, una mala imagen ante los consumidores. Por otra parte, el inventario no debe ser excesivo, ya que su costo de mantenimiento se elevaría.

La administración del inventario requiere dos decisiones fundamentales:

1. Definir la cantidad de pedido.

2. En qué momento debe hacerse un nuevo pedido.

La mayoría de los modelos de inventarios buscan definir algunos de los siguientes objetivos (Silver, 2008):

- Minimizar el costo incurrido en su manejo.

- Maximizar los beneficios económicos, incluyendo los ahorros por la compra de mayores volúmenes.
- Maximizar la tasa interna de retorno sobre la inversión.

- Definir una política adecuada de la administración del inventario.

En la mayoría de los casos, los modelos se han desarrollado para cumplir con el primero de dichos objetivos. Sus principios se apoyan en tres pilares básicos: el manejo de los inventarios es independiente de otras cuestiones administrativas; su función principal es la de amortiguar ante una demanda incierta; y la medida de desempeño usual es el costo del inventario.

Bixby (2013) propone 5 medidas para reducir el dinero desperdiciado en el manejo del inventario:

1. Reducir la variedad de productos, centrándose en aquellos que el cliente prefiere.

2. Reducir la variedad de tamaños del mismo producto, dejando los que agradan al cliente.

3. Detectar los productos que no se están moviendo o los que lo hacen lentamente.

4. Asignar una persona al manejo del inventario y pagarle según su desempeño.

5. Reducir las mermas por causas diversas.

Sin embargo, Chikán (2007) comenta que hay un cambio de paradigma respecto al manejo del inventario, que debe aplicarse por las empresas, a fin de seguir manteniendo su competitividad. Este nuevo paradigma se refiere a que los inventarios son parte integral de la cadena de valor, estrechamente relacionados con otras funciones de la organización, de modo que sean 
una herramienta estratégica para alcanzar el beneficio económico y la satisfacción del cliente; asimismo, las medidas de su desempeño deben basarse en su aportación para encontrar mejores soluciones para su clientela a diferencia de las que les brindan sus competidores. En otro estudio reciente realizado con 51 empresas húngaras, Chikán (2009) exploró la visión del papel que desempeñan en las organizaciones los inventarios. Encuentra que se ha dado un cambio gradual de paradigma, ocasionado por las circunstancias económicas actuales. Ahora los inventarios tienen una función estratégica más compleja, la cual se apoya en tres roles que deben cumplir:

I. Como creadores de valor.

2. Que den a la empresa flexibilidad.

3. Como un mecanismo de control.

Barry (2007) sugiere las siguientes métricas para el manejo del inventario: las tasas inicial y final de órdenes atendidas, la tasa de artículos devueltos y cancelaciones, la rotación del inventario y el margen de retorno sobre la inversión.

Este trabajo se realizó a partir de la información estadística basada en los registros históricos de la demanda y el tiempo de entrega, esto para determinar el impacto que la media o la varianza del tiempo de entrega, al igual que la de la demanda, tienen sobre el costo del inventario para dos artículos: el primero con demanda y tiempo de entrega con probabilidad normal y el segundo con distribución uniforme, de modo que se obtengan conclusiones que lleven a un mejor manejo del inventario.

\section{Revisión de la literatura}

En la administración de inventarios si la demanda y el tiempo de entrega del proveedor son inciertos, las organizaciones definen un stock de seguridad que les permita atender la demanda durante el tiempo de entrega, de modo que no se llegue a la situación de tener faltantes en el inventario que les signifique pérdida de ventas, así como una mala imagen ante los clientes, lo que hoy en día es esencial dada la gran competencia entre todos los sectores comerciales.

Fiom (2012) señala que el stock de seguridad es una protección contra la incertidumbre, que puede incluir variaciones en la demanda de los clientes, en el tiempo de entrega del proveedor y en la calidad de los productos.

Kanet et al. (2010) sugieren el uso de un stock de seguridad variable para ahorrar en la administración del inventario, con un valor mayor si la demanda o la oferta son más inciertas.

Van Kampen et al. (2010) afirman que las 2 medidas usuales para enfrentar la incertidumbre en la oferta y la demanda de un artículo son el stock de seguridad y la seguridad del tiempo de entrega. Sin embargo, estos fenómenos se han estudiado en condiciones aisladas, pasando por alto la incertidumbre de la oferta y la demanda. Encuentran para un sistema de productos múltiples mediante simulación, que si la incertidumbre está en la oferta, se maneje un tiempo de entrega con margen de seguridad. En caso de que se encuentre del lado de la demanda, es preferible manejar un stock de seguridad apropiado, en contraste con estudios previos para un artículo único. Si hay desconfianza en la información de la oferta y la demanda, sugieren que la mejor medida sea manejar un tiempo de entrega seguro.

Para artículos que se comportan con distribución normal, Eppen y Martin (1988) han encontrado errores en el cálculo del punto de reorden, por lo cual proponen un algoritmo para corregir estas fallas, que puede aplicarse en caso de que los parámetros en la demanda y el tiempo de entrega sean conocidos o desconocidos.

Ramesh (2009) señala que el pronóstico de la demanda es un elemento clave en el proceso de planeación para la manufactura de bienes de consumo envasados, de modo que se logre un buen nivel de servicio. Existen dos variables que intervienen para un buen pronóstico: la incertidumbre de la demanda y su variabilidad. Hay técnicas robustas para el manejo de la incertidumbre, pero no para la variabilidad. Para enfrentar este problema, Ramesh propone tomar en cuenta la voz del cliente en el proceso de planeación de la demanda.

Nasri et al. (2008) encontraron que invertir en la reducción de la varianza del tiempo de entrega produce ahorros en el costo del inventario.

Bolarín et al. (2009) comentan que la variabilidad de la demanda afecta al manejo de una cadena de suministro, ya que incrementa el efecto látigo en la cadena e impacta en los costos del inventario y en la tasa de faltantes.

King (2011) afirma que el manejo del inventario busca cumplir dos metas fundamentales: otorgar el nivel de servicio deseado por el cliente y que se realice a un costo mínimo. Estos objetivos deben balancearse para encontrar una solución apropiada. Señala que la variabilidad de la demanda del tiempo de entrega es la variable que más impacta esta decisión.

Paknejad et al. (1992) concluyen que la varianza del tiempo de entrega es más costosa que la varianza de la demanda. Por contraparte, Das (1975) asevera que la 
media del tiempo de entrega y no su varianza es lo que afecta en mayor medida al costo del inventario, mientras que Vinson (1972) afirma que la varianza del tiempo de entrega es de mayor importancia que su valor promedio o la variabilidad de la demanda para explicar el comportamiento del costo del inventario.

Hayya, Harrison y Chatfield (2009) plantean la posibilidad de desarrollar una relación del costo del inventario en función de la desviación estándar de la demanda del tiempo de entrega, para el caso de una demanda estacional y distribuida normalmente, con tiempo de entrega determinístico. Establecen que la media de la demanda del tiempo de entrega no tiene impacto significativo en el costo del inventario, pero sí la desviación estándar, de modo que si se desea reducir el costo, debe disminuirse esta variable.

Para un producto con tiempo de entrega exponencial, He (2009) encuentra que bajo el modelo de la cantidad económica de pedido con pedidos retroactivos, es la variabilidad del tiempo de entrega y no su valor promedio lo que impacta al costo del inventario.

Por su parte Gallego (2000) afirma que para un producto con distribución normal, la desviación estándar del tiempo de entrega afecta en mayor medida que su valor promedio a la demanda del tiempo de entrega, de modo que las organizaciones preferirán un tiempo de entrega más elevado, pero con menos variabilidad.

He et al. (2005) afirman que para el caso de un producto con demanda exponencial, el costo del inventario se ve afectado en mayor medida por la variabilidad del tiempo de entrega que por su valor promedio y esta relación de incremento es lineal con la desviación estándar del tiempo de entrega.

Kim et al. (2004) señalan que ni la cantidad económica de pedido, ni el costo óptimo del inventario se ven afectados por el tiempo de entrega y demuestran que para un nivel del límite superior, el incremento esperado del costo del inventario debido a un tiempo de entrega estocástico es lineal con la desviación estándar del tiempo de entrega.

Song (1994) establece que si el tiempo de entrega aumenta, esto genera un incremento en la demanda del tiempo de entrega y por consiguiente del stock de seguridad, aunque no necesariamente un costo óptimo del inventario más elevado. Si el tiempo de entrega tiene más variabilidad (mayor desviación estándar), habrá una demanda del tiempo de entrega mayor y un costo óptimo más alto. Esta variabilidad se ve afectada por la estructura de costos del inventario, concretamente la razón del costo de faltantes al de mantenimiento del inventario.
Con una demanda del tiempo de entrega normal, para un nivel de servicio entre $50 \%$ y un valor umbral máximo, si se reduce la desviación estándar del tiempo de entrega, se incrementa el punto de reorden, mientras que con una reducción de la media del tiempo de entrega, el punto de reorden disminuye (Chopra et al., 2004). Por lo tanto, si las compañías operan en este rango del nivel de servicio, la medida correcta para reducir el costo del inventario es disminuir la media del tiempo de entrega y no su variabilidad.

Fang et al. (2013) aseveran que no puede establecerse una conclusión contundente del impacto al reducir la media o la varianza del tiempo de entrega para incrementar la rentabilidad del manejo del inventario, ya que esta decisión depende si estos dos parámetros están o no correlacionados.

Mediante simulación aplicada a empresas del sector de transporte de carga, Dullaert y Zamparini (2013) buscan determinar el impacto de la media o la varianza del tiempo de entrega en el costo del inventario, concluyendo que una reducción de la variabilidad no necesariamente reduce los costos, de hecho podría incrementarlos, lo que depende de la distribución en la demanda del tiempo de entrega y del nivel de servicio meta que se tenga.

Para un artículo con tiempo de entrega con distribución de probabilidad gamma, afirman Wang y Hill (2006) que si el nivel de servicio se ubica entre 60 y 70\%, con una varianza mayor en el tiempo de entrega, el stock de seguridad primero se incrementa, que sería lo usual, pero después disminuye o permanece constante, lo que constituye un efecto recursivo.

Wang et al. (2010) derivaron fórmulas para estimar el punto de reorden y el stock de seguridad en caso que la demanda y el tiempo de entrega estén correlacionados, ya que los modelos tradicionales asumen que estas dos variables no se correlacionan, lo cual puede no ser válido, como en el caso de que un cliente importante haga pedidos de mayor volumen para satisfacer la demanda, o si la demanda va a la baja y el proveedor hace corridas de producción más pequeñas y tarda más en proveerlas.

Como se ha detallado, hay una vasta diversidad de opiniones del efecto que puede tener la media y la desviación estándar del tiempo de entrega y de la demanda en el costo del inventario y es precisamente lo que este estudio indaga.

\section{Desarrollo}

Este estudio pretende averiguar el impacto que la media o la desviación estándar del tiempo de entrega y de la demanda tienen en el costo del inventario, para el 
caso que los artículos tengan una distribución de la demanda del tiempo de entrega normal o uniforme.

Se asume que se conoce con base en registros históricos la información de la demanda, del tiempo de entrega y de los costos implicados en el manejo del inventario, como son el de colocar pedidos, mantener el inventario y el de los faltantes que surjan.

El costo total del inventario, $\mathrm{Ct}$, se calcula con la ecuación (1)

$C t=C p\left(\frac{D}{Q}\right)+C c\left(B+\frac{Q}{2}\right)+C f N f\left(\frac{D}{Q}\right)$

donde

$C t=$ costo total anual del inventario, $\$$ /año

$C p=$ costo de colocar un nuevo pedido, $\$ /$ pedido

$D=$ demanda anual de artículos, unidades/año

$Q=$ cantidad de pedido, unidades/pedido

$C c=$ costo anual de mantenimiento en el inventario, $\$$ / unidad

$B=$ existencias de seguridad, unidades

$C f=$ costo de cada faltante, $\$ /$ faltante

$N f=$ número promedio de faltantes, faltantes/pedido

El primer término de la ecuación se refiere al costo de hacer pedidos, el segundo al mantenimiento del inventario y el tercero a la aparición de faltantes.

La cantidad de pedido $Q$ se obtiene con la típica ecuación de Wilson sin considerar faltantes

$Q=\sqrt{\frac{2 C p D}{C c}}$

Donde todos los términos se definen previamente.

Por su parte, el punto de reorden PR, se obtiene con la ecuación (3)

$P R=\mu_{\mathrm{w}}+B$

Donde $\mu_{w}$ es la demanda promedio del tiempo de entrega, la cual se obtiene mediante la ecuación (4)

$\mu_{\mathrm{w}}=\mu_{\mathrm{x}} \mu_{\mathrm{y}}$

Aquí $\mu_{\mathrm{x}}$ es la demanda promedio diaria y $\mu_{\mathrm{y}}$ la media del tiempo de entrega en días.

Asimismo, la desviación estándar del tiempo de entrega se obtiene para la distribución normal con la siguiente ecuación $\sigma_{\mathrm{w}}=\sqrt{\mu_{\mathrm{y}} \sigma_{x}^{2}+\mu_{x}^{2} \sigma_{y}^{2}}$

donde

$\sigma_{x}=$ desviación estándar de la demanda, unidades/día

$\sigma_{\mathrm{y}}=$ desviación estándar del tiempo de entrega, días

Y para la distribución uniforme

$\sigma_{w}=\sqrt{\frac{\left(a_{w}-b_{w}\right)^{2}}{12}}$

donde $\alpha_{\mathrm{w}}$ y $\mathrm{b}_{\mathrm{w}}$ son los límites superior e inferior de la distribución uniforme para la demanda del tiempo de entrega, respectivamente.

El stock de seguridad se obtiene con la ecuación (7)

$B=Z \sigma_{w}$

Aquí $\mathrm{Z}$ es el número de desviaciones estandarizadas correspondiente al área de cada distribución de probabilidad, dada por el nivel de servicio meta.

Por otro lado, el nivel de servicio denotado como P (Z), se da por la siguiente relación de costos

$P(Z)=\frac{C f\left(\frac{D}{Q}\right)}{C c+C f\left(\frac{D}{Q}\right)}$

El numerador representa el costo de los faltantes y el denominador la suma de este costo y el de mantenimiento del inventario.

El número de faltantes $\mathrm{Nf}$, se aplica por la siguiente expresión matemática

$N f=\int_{P R}^{L S}(x-P R) f(x) d x$

donde

$\mathrm{x}=$ demanda del tiempo de entrega, unidades

$\mathrm{PR}=$ punto de reorden, unidades

LS = límite superior de la distribución de probabilidad, unidades

$\mathrm{f}(\mathrm{x})=$ función de probabilidad

Para el caso de la distribución normal se puede emplear la siguiente relación con funciones del software Excel 
$\mathrm{Nf}=-\mathrm{B}(1-\mathrm{DISTR} \cdot \mathrm{NORM}(\mathrm{Z}, 0,1,1))$

$+\sigma_{\mathrm{w}}($ DISTR.NORM $(Z, 0,1,0))$

Y para la distribución uniforme, el número de faltantes se calcula con la ecuación (11)

$$
N f=\frac{\left(a_{w}-P R\right)^{2}}{2\left(a_{w}-b_{w}\right)}
$$

Donde $\alpha_{\mathrm{w}} \mathrm{y} \mathrm{b}_{\mathrm{w}}$ se definen previamente.

Entonces se efectúan cálculos del costo del inventario con valores de un caso base para cada distribución de probabilidad y se varían luego los valores de la media y desviación estándar del tiempo de entrega, así como la desviación estándar y la media de la demanda, para ver cuál de estas variables afecta en mayor medida al costo, además de confirmar si la relación matemática entre el costo del inventario es lineal con cada variable y si este comportamiento cambia con el valor del nivel de servicio meta planeado.

\section{Discusión y análisis de resultados}

Para el artículo con distribución normal de la demanda del tiempo de entrega, los datos del caso base son los que se presentan en la tabla 1 .

Tabla 1. Datos del caso base para el artículo con distribución normal

\begin{tabular}{cc}
\hline Parámetro & Valor \\
\hline $\begin{array}{c}\text { Media de la demanda, unidades/día } \\
\text { día }\end{array}$ & 100 \\
Desviación estándar de la demanda, unidades/ & 16 \\
Media del tiempo de entrega, días & 8 \\
Desviación estándar del tiempo de entrega, días & 2 \\
Costo de mantenimiento del artículo, \$/(año) & 45.00 \\
$\quad$ (unidad) & 800.00 \\
Costo de colocar un pedido, \$/pedido & 60.00 \\
\hline Costo de faltantes, \$/(pedido)(unidad)
\end{tabular}

Fuente: Elaboración propia

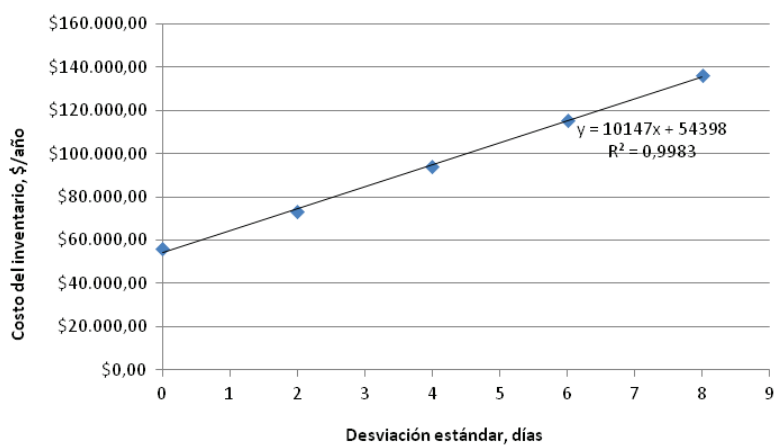

Con estos datos se calcula la cantidad de pedido con base en la ecuación (2) para obtener

$Q=\sqrt{\frac{2(800)(100)(365)}{45}}=1,139.2$ unidades / pedido

El nivel de servicio es

$$
P(Z)=\frac{(60)\left(\frac{(100)(365)}{1139.2}\right)}{45+(60)\left(\frac{(100)(365)}{1139.2}\right)}=0.977
$$

Cuyo valor correspondiente de Z es 1.998 .

La demanda del tiempo de entrega es conforme a la ecuación (4)

$\mu_{w}=(100)(8)=800$ unidades

Y la desviación estándar (ecuación 5)

$\sigma_{\mathrm{w}}=\sqrt{(8)(16)^{2}+(100)^{2}(2)^{2}}=205.06$ unidades

Con esto el valor de las existencias de seguridad B, es

$B=(1.998)(205.06)=409.66$ unidades

Y el número de faltantes, conforme a la ecuación (10)

$\mathrm{Nf}=-(409.66)(1-D I S T R \cdot N O R M(1.998,0,1,1))+(205.06)$

$($ DISTR.NORM $(1.998,0,1,0))=1.75$ faltantes/pedido

Donde el costo del inventario para el caso base es

$$
\begin{aligned}
& C t=(800)\left(\frac{(100)(365)}{1,139.2}\right)+(45)\left(409.66+\frac{1,139.2}{2}\right) \\
& +(60)(1.75)\left(\frac{(100)(365)}{1,139.2}\right)=73,070.69 \$ / \text { año }
\end{aligned}
$$

Figura 1. Variación del costo del inventario con la desviación estándar del tiempo de entrega

Fuente: Elaboración propia 
Si se repiten estos cálculos para valores de la desviación estándar del tiempo de entrega $\sigma_{\mathrm{y}}$ comprendidos en un rango de cero a 8 unidades, se obtienen los resultados de la figura 1.

En el gráfico se incluye la ecuación lineal del ajuste y el coeficiente de determinación $\mathrm{R}^{2}$ que señala la bondad del ajuste lineal entre el costo del inventario y la desviación estándar, que es casi perfecto al resultar en un valor de 0.998. La ecuación muestra que por cada día que se incremente la desviación estándar del tiempo de entrega, el costo del inventario aumenta un poco más de 10 mil pesos anuales.

Si ahora se deja constante la desviación estándar del tiempo de entrega, en su valor original del caso base de 2 días y se hace variar la media del tiempo de entrega en un rango de 4 a 16 días, se obtiene el gráfico de la figura 2, en el cual se puede ver que el ajuste lineal entre las variables es perfecto y con la pendiente de la ecuación se define aproximadamente que por cada día que se incremente el promedio del tiempo de entrega, el costo aumenta 66 pesos.

Con esto se afirma que el costo del inventario se ve impactado en mayor medida por la desviación estándar que por el promedio del tiempo de entrega.

Si se repiten los cálculos variando a la vez las medias y desviaciones estándar de la demanda y el tiempo de entrega, mediante regresión lineal múltiple, se obtienen los resultados que se sintetizan en la tabla 2.

Tabla 2. Valores del ajuste de la regresión

\begin{tabular}{cccc}
\hline Variable & $\begin{array}{c}\text { Beta no } \\
\text { estandarizada }\end{array}$ & $\begin{array}{c}\text { Beta } \\
\text { estandarizada }\end{array}$ & $\begin{array}{c}\text { Significancia } \\
\text { estadística }\end{array}$ \\
\hline$\mu_{\mathrm{x}}$ & 445.68 & 0.651 & 0.000 \\
$\alpha_{\mathrm{x}}$ & 150.44 & 0.035 & 0.493 \\
$\mu_{\mathrm{y}}$ & 204.52 & 0.030 & 0.561 \\
$\sigma_{\mathrm{y}}$ & 14741.56 & 0.703 & 0.000 \\
\hline
\end{tabular}

Fuente: Elaboración propia

El coeficiente de determinación de la regresión $\mathrm{R}^{2}$ es de 0.92 , que indica un buen ajuste. Las dos variables que

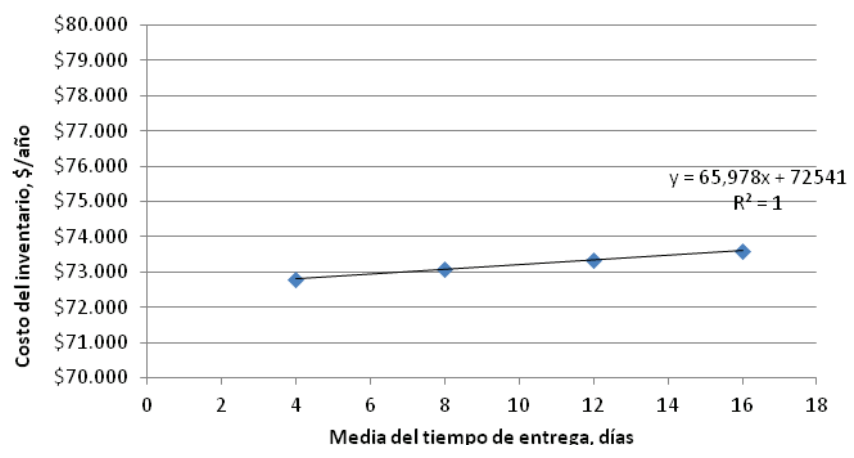

resultan significativas (alfa menor de 0.05 ) son la media de la demanda y la desviación estándar del tiempo de entrega, lo cual se confirma con los valores de las betas estandarizadas.

Con esto se concluye que es la desviación estándar del tiempo de entrega y no su valor promedio la variable que más afecta al costo del inventario. Además la demanda promedio también impacta al costo del inventario.

Estos cálculos se hicieron para un nivel de servicio de 0.977 , si se repiten para valores de 0.502 y 0.997 , el impacto de las 4 variables sobre el costo del inventario no cambia.

Si se hace el cálculo para el artículo con distribución uniforme, los datos de la demanda, el tiempo de entrega y los costos son los que se anotan en la tabla 3.

Tabla 3. Datos del caso base para el artículo con distribución uniforme

\begin{tabular}{lc}
\hline \multicolumn{1}{c}{ Parámetro } & Valor \\
\hline Media de la demanda, unidades/día & 120 \\
Límites inferior y superior de la demanda, unidades/ & 90 y \\
día & 150 \\
$\begin{array}{l}\text { Desviación estándar de la demanda, unidades/día } \\
\text { Media del tiempo de entrega, días }\end{array}$ & 17.32 \\
$\begin{array}{l}\text { Límites inferior y superior del tiempo de entrega, } \\
\text { días }\end{array}$ & 5 y 11 \\
Desviación estándar del tiempo de entrega, días & 1.73 \\
$\begin{array}{l}\text { Costo de mantenimiento del artículo, \$/(año) } \\
\text { (unidad) }\end{array}$ & 140.00 \\
Costo de colocar un pedido, \$/pedido & 800.00 \\
Costo de faltantes, \$/(pedido)(unidad) & 100.00 \\
\hline
\end{tabular}

Fuente: Elaboración propia

Las desviaciones estándar de la demanda y del tiempo de entrega se calcularon con la ecuación (6) y Q con la ecuación (2)

$Q=\sqrt{\frac{2(800)(120)(365)}{140}}=707.5$ unidades / pedido

Figura 2. Cambio del costo del inventario con la media del tiempo de entrega

Fuente: Elaboración propia 
El nivel de servicio es

$$
P(Z)=\frac{(100)\left(\frac{(120)(365)}{707.5}\right)}{140+(100)\left(\frac{(120)(365)}{707.5}\right)}=0.978
$$

Que corresponde a un valor de Z de 1.915.

La demanda del tiempo de entrega se aplica conforme a la ecuación (4)

$\mu_{w}=(120)(8)=960$ unidades

Y la desviación estándar (ecuación 6)

$\sigma_{w}=\sqrt{\frac{(1650-450)^{2}}{12}}=346.41$ unidades

Las existencias de seguridad se dan mediante a la ecuación (7)

$B=(1.915)(346.41)=663.46$ unidades

Y el punto de renovación del pedido es

$P R=960+663.46=1,623.46$ unidades

El número de faltantes se calcula con la ecuación (11)

$N f=\frac{(1650-1623.46)^{2}}{2(1650-450)}=0.29$ faltantes/pedido

Donde el costo del inventario es

$$
\begin{aligned}
& C t=(800)\left(\frac{(120)(365)}{707.5}\right)+(140)\left(663.46+\frac{707.5}{2}\right) \\
& +(100)(0.29)\left(\frac{(120)(365)}{707.5}\right)=193,752.81 \$ / \text { año }
\end{aligned}
$$

Si se repiten los cálculos para diversos valores de la desviación estándar del tiempo de entrega $\sigma_{\mathrm{y}}$ comprendidos en un rango de cero a 2.3 unidades, se obtiene el gráfico de la figura 3.

El ajuste lineal es perfecto con una pendiente de 35,721 , que es lo que aumentará el costo del inventario por incrementar la desviación estándar del tiempo de entrega en un día.

Si se hace lo propio para determinar el impacto que sobre el costo del inventario tiene la media del tiempo de entrega, se produce el gráfico de la figura 4.

El ajuste lineal también resulta perfecto $\left(R^{2}=1\right)$ y el costo aumenta poco más de 4 mil pesos por un incremento de un día en el tiempo de entrega.

Si se hace algo similar modificando las 4 mismas variables que en el caso de la distribución normal, las medias y desviaciones estándar de la demanda y el tiempo de entrega, se obtienen los resultados de la tabla 4.

Tabla 4. Valores del ajuste de la regresión

\begin{tabular}{cccc}
\hline Variable & $\begin{array}{c}\text { Beta no } \\
\text { estandarizada }\end{array}$ & $\begin{array}{c}\text { Beta } \\
\text { estandarizada }\end{array}$ & $\begin{array}{c}\text { Signif. } \\
\text { estadística }\end{array}$ \\
\hline$\mu_{\mathrm{x}}$ & 554.10 & 0.535 & 0.000 \\
$\alpha_{\mathrm{x}}$ & 3217.52 & 0.449 & 0.000 \\
$\mu_{\mathrm{y}}$ & 6174.99 & 0.398 & 0.000 \\
$\sigma_{\mathrm{y}}$ & 53794.17 & 0.559 & 0.000 \\
\hline
\end{tabular}

Fuente: Elaboración propia

El coeficiente $R^{2}$ resultó de 0.96 que indica un buen ajuste. En este caso las 4 variables son significativas, en mayor medida la desviación estándar del tiempo de entrega, seguida de la media de la demanda.

Nuevamente la desviación estándar del tiempo de entrega impacta más que su media al costo del inventario.

Si se repiten estos cálculos para niveles de servicio de 0.998 o 0.516 , las 4 variables resultan estadísticamente significativas, solo que en ambos casos la variable con la beta estandarizada más alta es ahora la media de la demanda, seguida por la desviación estándar del tiem-

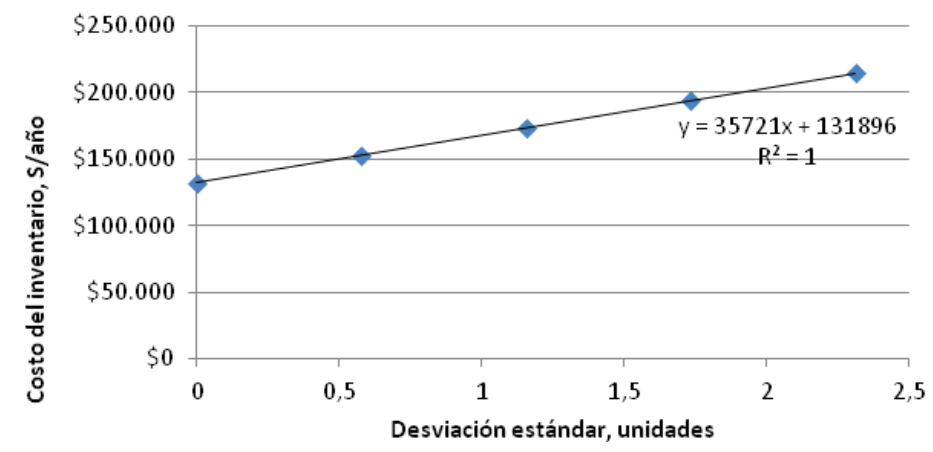

Figura 3. Cambio del costo del inventario con la desviación estándar del tiempo de entrega

Fuente: Elaboración propia 


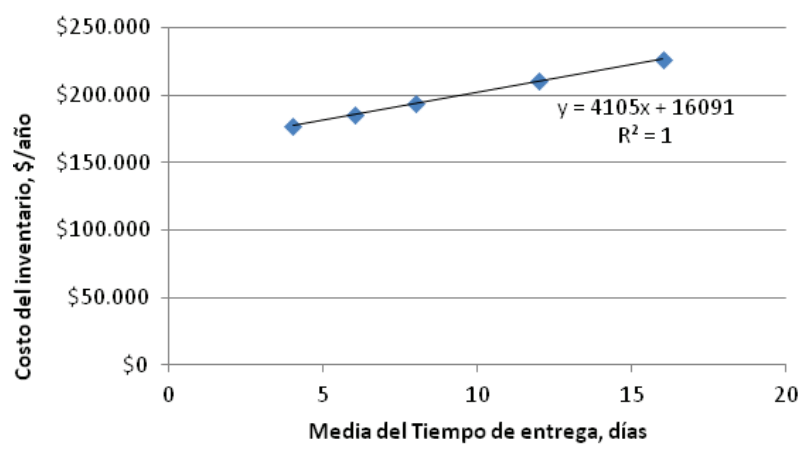

po de entrega y la que queda con la beta menor es la media del tiempo de entrega.

\section{Conclusiones}

Se ha visto que para ambas distribuciones de probabilidad, la desviación estándar del tiempo de entrega tiene mayor impacto en el costo del inventario que su media, lo que concuerda con los hallazgos de varios investigadores (Gallego, 2000; Hayya et al., 2009; King, 2011; Nasri et al., 2008; Van Kampen et al., 2010) y contrasta con otros como Das (1975).

Asimismo, en concordancia con lo afirmado por Kim y colaboradores (2004), el costo del inventario varía linealmente con la desviación estándar del tiempo de entrega, tal y como se mostró en la figura 1 para el producto con distribución normal, y en la figura 3 para el de distribución uniforme.

Si la incertidumbre de la demanda del tiempo de entrega es alta, se requiere de un mayor stock de seguridad y se incurre en un costo del inventario más elevado, lo que concuerda con lo afirmado por Kanet y colaboradores (2010).

Para el artículo con distribución normal, la desviación estándar del tiempo de entrega tiene mayor impacto en el costo del inventario que la desviación estándar de la demanda, en concordancia con lo señalado por Paknejad y colaboradores (1992). Sin embargo, para el producto con distribución uniforme esto se cumple solo en el primer caso del nivel de servicio (97.8\%), no así en los otros dos casos analizados, cuyos niveles de servicio fueron 99.8 y $51.6 \%$, por lo cual se puede decir que esta afirmación queda sujeta al valor del nivel de servicio que se tenga.

Asimismo, en todos los casos analizados la desviación estándar del tiempo de entrega es una variable de mayor importancia que su valor promedio, o que la desviación estándar de la demanda, lo que confirma los hallazgos de Vinson (1972).
Figura 4. Cambio del costo del inventario con la media del tiempo de entrega

Fuente: Elaboración propia

Contrariamente a lo afirmado por Song (1994), el efecto de la desviación estándar no depende de la estructura de costos, ya que en todos los casos al aumentar esta variable, el costo del inventario sube.

De igual forma, para cualquier nivel de servicio, si la desviación estándar del tiempo de entrega aumenta, el punto de reorden y el costo del inventario también lo hacen, de modo que en contraste a lo afirmado por Chopra y colaboradores (2004), la variable más importante para cumplir con un nivel de servicio meta a un costo mínimo es la desviación estándar del tiempo de entrega y no su valor promedio.

Este estudio también afirma que contrario a lo señalado por Fang y colaboradores (2013), la variabilidad del tiempo de entrega en todos los casos analizados es más importante que su media y no depende de si ambas variables están correlacionadas.

Para las dos distribuciones de probabilidad vistas (normal y uniforme) y para cualquier nivel de servicio, si la variabilidad del tiempo de entrega disminuye, esto lleva a una reducción del costo del inventario, lo que se contrapone a lo afirmado por Dullaert y Zamparini (2013), que esto depende de la distribución de probabilidad y el nivel de servicio manejado.

De esta investigación queda claro que el impacto de las 4 variables sobre el costo del inventario depende de la distribución de probabilidad que se tenga para la demanda del tiempo de entrega; por otro lado, las 2 variables con mayor efecto sobre el costo son la desviación estándar del tiempo de entrega y el promedio de la demanda de artículos para ambas distribuciones de probabilidad.

La diferencia entre ambas distribuciones es que para la normal no han resultado significativas la media del tiempo de entrega y la desviación estándar de la demanda, en cambio, para el caso del artículo con distribución uniforme las 4 variables son significativas y en dos de los tres niveles de servicio analizados resultó con mayor impacto en el costo del inventario la media 
de la demanda en comparación con la desviación estándar del tiempo de entrega.

Con esto queda claro que si se desea manejar el inventario de manera eficiente para cumplir con el nivel de servicio deseado a un costo mínimo del inventario, las dos variables que deben emplearse son la media de la demanda de artículos y la desviación estándar del tiempo de entrega. Como la media de la demanda no es deseable que disminuya, porque representaría menores volúmenes de venta, debe tenerse en cuenta la selección de proveedores con las organizaciones que cuenten con un tiempo de entrega razonable y variabilidad mínima.

\section{Referencias}

Barry C. The best of inventory. Multichannel Merchant, volumen 3 (número 1), 2007: 1-45.

Bixby B. Five ways to stop wasting cash in inventory: industry insights. CSDecisions, volume 21, 2013.

Bolarín F.C., Frutos A.G., McDonell L.R. The influence of lead time variability on supply chains costs: analysis of its impact on the bullwhip effect. Journal of Supply Chain Management, volumen 6 (números 3/4), 2009: 15-26.

Chikán A. The new role of inventories in business: Real world changes and research consequences. International Journal of Production Economics, volumen 108, 2007: 54-62.

Chikán A. An empirical analysis of managerial approaches to the role of inventories. International Journal of Production Economics, volumen 118 (número 1), 2009: 131-135.

Chopra S., Reinhardt G., Dada M. The effect of lead time uncertainty on safety stocks. Decision Sciences, volumen 35 (número 1), 2004: 1-24.

Das C. Effect of lead time on inventory: A static analysis. Operational Research Quarterly, volumen 26 (número 2), 1975: 273-282.

Dullaert W. y Zamparini L. The impact of lead time reliability in freight transport: A logistics assessment of transport economics findings. Transportation Research, Part E, volumen 49 (número 1), 2013: 190-200.

Eppen G.D. y Martin R.K. Determining safety stock in the presence of stochastic lead time and demand. Management Science, volumen 34 (número 11), 1988: 1380-1390.

Fang X., Zhang Ch., Robb D.J., Blackburn J.D. Decision support for lead time and demand variability reduction. Omega, volumen 41 (número 2), 2013: 390-396.

Fiom S.G. Effective and efficient use of safety or buffer stock. Operations Management, volumen 5, 2012: 27-31.

Gallego G. Variability of lead time demand and the protection period. IEOR4000: Production Management, volumen 1-3, 2000.
Hayya J.C., Harrison T.P., Chatfield D.C. Exploring the structural properties of the $(\mathrm{D}, 0)$ inventory model. International Journal of Production Research, volumen 47 (número 10), 2009: 27672783.

He X.J. The Impact of Stochastic Lead Time: the Mean or the Variance. Proceedings of the International Multi Conference of Engineers and Computer Scientists, Vol. II, Hong Kong, 1-5, 2009.

He X.J., Kim J.G., Hayya J.C. The cost of lead-time variability: the case of the exponential distribution. International Journal of Production Economics, volumen 97 (número 2), 2005: 113-122.

Kanet J.J., Gorman M.F., Stoblëin M. Dynamic planned safety stocks in supply networks. International Journal of Production Research, volumen 48 (número 22), 2010: 6859-6880.

Kim J.G., Sun D., He X.J., Hayya J.C. The (s, Q) Inventory model with erlang lead time and deterministic demand. Naval Research Logistics, volumen 51 (número 6), 2004: 906-923.

King P.L. Crack the code. understanding safety stock and mastering its equations. APICS Magazine, julio/agosto 2011: 33-36.

Nasri F., Paknejad J., Affisco J.F. Investing in lead-time variability reduction in a quality-adjusted inventory model with finiterange stochastic lead-time. Journal of Applied Mathematics and Decision Sciences, volumen 2008 (número 1), 2008: 1-13.

Paknejad M.J., Nasri F., Affisco J.F. Lead-time variability reduction in stochastic inventory models. European Journal of Operations Research, volumen 62 (número 3), 1992: 311-322.

Ramesh V. Effectively managing demand variability in CPG Industry with focus on CPG manufacturer. Infosys, white paper, 1-6, 2009 [fecha de consulta: 15 de noviembre de 2014]. Disponible en: http://www.infosys.com.

Silver E.A. Inventory management: An overview, Canadian publications, practical applications and suggestions for future research. Information Systems and Operations Research, volumen 46 (número 1), 2008: 15-28.

Song J. The effect of lead time uncertainty in a simple stochastic inventory model. Management Science, volumen 40 (número 5), 1994: 603-613.

Van-Kampen T.J., Van-Donk D.P., Van-Der Z.D. Safety stock or safety lead time: coping with unreliability in demand and supply. International Journal of Production Research, volumen 48 (número 24), 2010: 7463-7481.

Vinson C. The cost of ignoring lead-time unreliability in inventory theory. Decision Sciences, volumen 3 (número 2), 1972: 87-105.

Wang P. y Hill J.A. Recursive behavior of safety stock reduction: The effect of lead-time uncertainty. Decision Sciences, volumen 37 (número 2), 2006: 285-290.

Wang P., Zinn W., Croxton K.L. Sizing inventory when lead time and demand are correlated. Production and Operations Management, volumen 19 (número 4), 2010: 480-484. 


\author{
Este artículo se cita: \\ Citación estilo Chicago \\ Izar-Landeta, Juan Manuel, Carmen Berenice Ynzunza-Cortés, Ar- \\ turo Castillo-Ramírez, Raúl Hernández-Molinar. Estudio compara- \\ tivo del impacto de la media y varianza del tiempo de entrega y de \\ la demanda en el costo del inventario. Ingeniería Investigación y \\ Tecnología, XVII, 03 (2016): 371-381.

\section{Citación estilo ISO 690} \\ Izar-Landeta J.M., Ynzunza-Cortés C.B., Castillo-Ramírez A., Her- \\ nández-Molinar R. Estudio comparativo del impacto de la media y \\ varianza del tiempo de entrega y de la demanda en el costo del \\ inventario. Ingeniería Investigación y Tecnología, volumen XVII \\ (número 3), julio-septiembre 2016: 371-381.
}

\section{Semblanzas de los autores}

Juan Manuel Izar-Landeta. Doctor en administracion, SNI nivel I, ha publicado 13 libros, 4 capitulos de libros, cuenta con más de 80 artículos científicos y de divulgación, así como la participación en más de 65 ponencias en congresos nacionales e internacionales. Sus áreas de investigación son: Pymes, ingeniería industrial y pensiones y jubilaciones. Sus áreas de docencia son: estadística, finanzas, ingeniería económica, modelos cuantitativos para toma de decisiones y gestión y evaluación de proyectos.

Carmen Berenice Ynzunza-Cortés. Es doctora en administracion, SNI nivel I. Sus líneas de investigacion son: estrategia competitiva, recursos y capacidades organizacionales y desempeño organizacional. Tiene experiencia laboral de 15 años en la industria, ocupando puestos de gerencia de administración y finanzas. Es docente desde hace 18 años a nivel medio superior y superior. Ha publicado más de 20 articulos en revistas indizadas y un gran número de ponencias en congresos nacionales e internacionales

Arturo Castillo-Ramírez. Es maestro en planeacion y sistemas por la UASLP, donde labora como profesor investigador, es coordinador de acreditaciones y miembro del consejo técnico consultivo de la Facultad de Ingeniería con 22 años de experiencia docente. Es evaluador nacional de CACEI y miembro de la Sociedad Mexicana de Ingeniería Mecánica (SOMIM). Ha escrito un artículo en revista arbitrada, 2 apuntes para materias del Posgrado de Planeación y Sistemas y 4 publicaciones en memorias de congresos nacionales e internacionales de ACACIA y SOMIM.

Raúl Hernández-Molinar. Es doctor en ciencias por la Universidad de Tulane. Las áreas de interés en docencia e investigación son: teoría confiabilidad, teoría del valor extremo, análisis de riesgos, control de calidad industrial, diseño de experimentos, modelos no paramétricos, regresión lineal, sistemas de gestión de la calidad y el uso de la inferencia estadística en aplicaciones de la vida real. Ha participado como instructor en educación continua y como consultor para diferentes empresas en México y en el extranjero 